Conclusions: There is a direct correlation between the markers of inflammation and the evolution of carotid atheromatosis in patients with rheumatoid arthritis. The intima-medium thickness index is a paraclinical feature useful in assessing cardiovascular risk in patients with rheumatoid arthritis.

Biological therapy, by controlling the underlying disease, also improves cardiovascular prognosis. Treatment with anti-TNF-alpha makes good control, together with lipid-lowering and antihypertensive medication of associated cardiovascular pathology, in patients with rheumatoid arthritis.

Disclosure of Interest: None declared

DOI: 10.1136/annrheumdis-2018-eular.5109

\section{THU0108 PATIENT REPORTED OUTCOME MEASURES FOLLOWING KNEE AND HIP REPLACEMENT FOR INDIVIDUALS WITH RHEUMATOID ARTHRITIS: EVIDENCE FROM ROUTINELY-COLLECTED DATA}

E. Burn ${ }^{1}$, N.K. Arden ${ }^{1}$, C.J. Edwards ${ }^{2}$, C. Cooper ${ }^{1}$, D. Murray ${ }^{1}$, R. PinedoVillanueva ${ }^{1}$, D. Prieto-Alhambra ${ }^{1} .{ }^{1}$ NDORMS, University of Oxford, Oxford; ${ }^{2} \mathrm{NIHR}$ Clinical Research Facility, University Hospital Southampton, Southampton, UK

Background: Most estimates of patient-reported outcome measures (PROMs) following total knee replacement (TKR) and total hip replacement (THR) are from individuals with osteoarthritis (OA). It is not well-known whether individuals with rheumatoid arthritis $(\mathrm{RA})$ achieve similar results.

Objectives: To assess the association between $\mathrm{RA}$, relative to $\mathrm{OA}$, with 1) postoperative PROMs and 2) the change between pre- and post-operative PROMs for TKR and THR.

Methods: PROMs for TKR and THR between 2009 and 2015 identified using Hospital Episode Statistics (HES), with diagnosis of RA or OA identified in the Clinical Practice Research Datalink (CPRD). The condition-specific Oxford Knee Score/Oxford Hip Score (OKS/OHS), with pain and function subscales, and EuroQol 5-dimension (EQ-5D) overall quality of life were collected prior to and six months following surgery. OKS/OHS ranges from 0 to 48 , OKS/OHS function from 0 to 20, OKS/OHS pain from 0 to 28, and EQ-5D from -0.5 to 1 , with higher scores being better for each. The effect of RA, relative to OA, on post-operative PROMs was estimated using multivariable linear regressions, which adjusted for age, gender, comorbidities (measured by the Charlson score) and socioeconomic status (measured by IMD Quintile). The effect of RA, relative to OA, on change in $\mathrm{PROMs}$ was assessed by adding pre-operative scores as an explanatory factor in the models.

Results: 2212 (2,070 OA and 142 RA) and 2128 (2,030 OA and 98 RA) individuals informed the analyses of TKR and THR, respectively. A diagnosis of RA, relative to OA, was associated with a one-point lower post-operative OKS and a 3point lower OHS, although only the latter difference was significant. These lower estimates of post-operative OKS/OHS were primarily due to significantly lower scores on the function subscales. The estimated change in OKS/OHS, after accounting for pre-operative scores, was similar for those with RA and OA. RA was also associated with a 0.1 lower post-operative EQ-5D, which was significant for both TKR and THR. The expected change in EQ-5D remained significantly lower for those with RA. The effect of diagnosis from the models is summarised in table 1 , with estimated change in OKS/OHS shown in figure 1.

Abstract THU0108 - Table 1. Effect of RA, relative to OA, on 1) total post-operative scores and 2) change in scores. Estimated coefficients with 95\% confidence intervals. Significan differences in bold.

\begin{tabular}{|c|c|c|c|c|}
\hline & TKR & & THR & \\
\hline $\mathrm{OKS} / \mathrm{OHS}$ (0 to 48 ) & $\begin{array}{c}\text { Total } \\
-1.33(-2.92 \text { to } \\
0.27)\end{array}$ & $\begin{array}{c}\text { Change } \\
-0.07(-1.59 \text { to } \\
1.46)\end{array}$ & $\begin{array}{c}\text { Total } \\
-2.80(-4.43 \text { to } \\
-1.18)\end{array}$ & $\begin{array}{c}\text { Change } \\
-1.57(-3.15 \text { to } \\
0.01)\end{array}$ \\
\hline $\begin{array}{l}\text { OKS/OHS function } \\
(0 \text { to } 20)\end{array}$ & $\begin{array}{c}-1.22(-1.90 \text { to } \\
-0.54)\end{array}$ & $\begin{array}{c}-0.50(-1.13 \text { to } \\
0.14)\end{array}$ & $\begin{array}{c}-1.91(-2.75 \text { to } \\
-1.07)\end{array}$ & $\begin{array}{c}-0.97(-1.76 \text { to } \\
-0.18)\end{array}$ \\
\hline $\begin{array}{l}\text { OKS/OHS pain ( } 0 \text { to } \\
28 \text { ) }\end{array}$ & $\begin{array}{c}-0.13(-1.12 \text { to } \\
0.87)\end{array}$ & $\begin{array}{c}0.34(-0.63 \text { to } \\
1.30)\end{array}$ & $\begin{array}{c}-0.89(-1.80 \text { to } \\
0.02)\end{array}$ & $\begin{array}{c}-0.66(-1.56 \text { to } \\
0.25)\end{array}$ \\
\hline EQ-5D ( -0.5 to 1$)$ & $\begin{array}{c}-0.12(-0.16 \text { to } \\
-0.08)\end{array}$ & $\begin{array}{c}-0.09(-0.13 \text { to } \\
-0.06)\end{array}$ & $\begin{array}{c}-0.12(-0.17 \text { to } \\
-0.08)\end{array}$ & $\begin{array}{c}-0.08(-0.13 \text { to } \\
-0.04)\end{array}$ \\
\hline
\end{tabular}

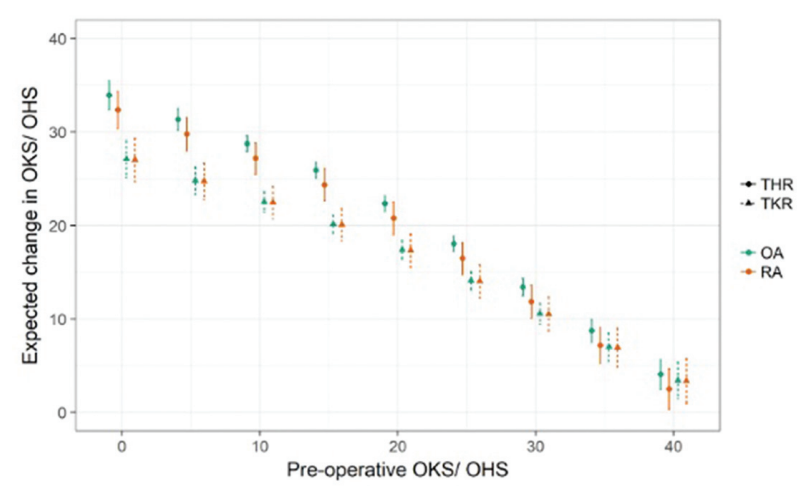

Abstract THU0108 - Figure 1. Estimated effect of diagnosis on expected change in OKS/ $\mathrm{OHS}$ by pre-operative score

Conclusions: Individuals with RA undergoing TKR and THR appear to achieve similar improvements in condition-specific scores as those with $\mathrm{OA}$, although the gain in function may be slightly less. The gain in overall quality of life is less for those with RA, however, which is likely due to the systemic nature of the disease. Disclosure of Interest: E. Burn: None declared, N. Arden Consultant for: Fresh fields Bruckhaus Deringer, Bioventus, Flexion, Merck, and Regeneron, C Edwards: None declared, C. Cooper: None declared, D. Murray Grant/research support from: Zimmer Biomet, Consultant for: Zimmer Biomet, R. Pinedo-Villa nueva: None declared, D. Prieto-Alhambra Grant/research support from: Amgen, Servier and UCB Biopharma, and non-financial support from Amgen DOI: 10.1136/annrheumdis-2018-eular.3753

\section{THU0109 AUTOANTIBODY PROFILING FOR RESPONSE TO BARICITINIB IN PATIENTS WITH RHEUMATOID ARTHRITIS AND NO OR LIMITED EXPOSURE TO METHOTREXATE}

L. Martinez Gamboa ${ }^{1}$, H. Bang ${ }^{2}$, M. Issa ${ }^{3}$, S. Zuckerman ${ }^{3}$, A. Cardoso ${ }^{3}$, T. Holzkaemper ${ }^{3}$, F. De Leonardis ${ }^{3}$, I. de la Torre ${ }^{3}, Y$. Tanaka ${ }^{4}$, E. Feist ${ }^{1}$. ${ }^{1}$ CharitéUniversitätsmedizin Berlin, Berlin; ${ }^{2}$ Orgentec Diagnostika GmbH, Mainz, Germany; ${ }^{3}$ Eli Lilly and Company, Indianapolis, USA; ${ }^{4}$ University of Occupational and Environmental Health, Kitakyushu, Japan

Background: Posttranslational antigen modification plays a role in the pathogenesis of rheumatoid arthritis (RA). The associated autoantibodies are considered unfavourable prognostic factors with respect to disease severity and radiographic outcome. Limited information is available for different serotypes and prediction of treatment response to Janus kinase inhibitors including baricitinib (BARI).

Objectives: To clarify whether fine profiling of baseline autoantibodies against different modified isoforms of vimentin correlate with clinical or radiographic outcome in patients (pts) with active RA and no or limited prior DMARD treatment who initiate treatment with BARI.

Methods: Baseline sera samples from DMARD-naïve pts participating in the randomised, active comparator-controlled $\mathrm{Ph} 3$ trial RA-BEGIN ${ }^{1}$ [methotrexate (MTX) $n=210$, BARI 4 mg (mono) $n=159$ and BARI 4-mg+MTX (combo) $n=215$ ] were investigated for autoantibody class $(\lg G, \lg M$ and $\lg A$ ) reactivity towards vimentin (V), modified by citrullination (MCV), carbamylation (Carb) or acetylation (Acct), using enzyme-linked immunosorbent assay. Pts were stratified according to negative $(<20 \mathrm{U} / \mathrm{ml})$, low-positive $(20-60 \mathrm{U} / \mathrm{ml})$ and high-positive $(>60 \mathrm{U} / \mathrm{ml}$, 3fold above the ULN) antibody titre or with respect to the antibody titre tertiles. Change in clinical outcomes (DAS28-CRP, CDAI) and radiographic progression (modified Total Sharp Score) were analysed from baseline to Week 24 using modified last observation carried forward and linear extrapolation, respectively, by baseline autoantibody subclass and titre. 
Results: Comparison of least squares mean difference in clinical scores showed more consistent improvement in pts treated with BARI combo vs MTX irrespective of baseline autoantibody subclass and titre. In general, pts with low-titre antiCarbV, AcctV and MCV subclass antibodies showed numerically less improvement in most of the analyses under BARI mono vs MTX compared to BARI combo vs MTX although for anti-CarbV subclass seronegative pts, no significant differences were found in the clinical response between BARI mono vs MTX. Furthermore, anti-MCV $\lg \mathrm{A}$ and $\lg \mathrm{M}$ as well as anti-CarbV $\lg \mathrm{A}$ negative status was also associated with significant reduction of radiographic progression in pts treated with BARI combo vs MTX. For seropositive pts, response to treatment with BARI mono or combination therapy was higher in pts with highest titres of anti-MCV and anti-CarbV. However, a significant difference with respect to radiographic progression was detectable only for BARI combo vs MTX. By stratifying pts according to their antibody profile, these observed radiographic differences were achieved in the anti-MCV and anti-CarbV IgG high-positive as well as anti-CarbV IgM lowpositive pts.

Conclusions: In these exploratory analyses, seropositive pts with high titres of anti-MCV and anti-CarbV at baseline showed better responses to BARI mono or combo vs MTX for composite scores, and to BARI combo in structural progression outcomes.

\section{REFERENCE:}

[1] Fleischmann R, et al. Arthritis Rheumatol 2017;69(3):506-517.

Disclosure of Interest: L. Martinez Gamboa Grant/research support from: Eli Lilly and Company, H. Bang: None declared, M. Issa Shareholder of: Eli Lilly and Company, Employee of: Eli Lilly and Company, S. Zuckerman Employee of: Former employee of Eli Lilly and Company, A. Cardoso Shareholder of: Eli Lilly and Company, Employee of: Eli Lilly and Company, T. Holzkaemper Shareholder of: Eli Lilly and Company, Employee of: Eli Lilly and Company, F. De Leonardis Shareholder of: Eli Lilly and Company, Employee of: Eli Lilly and Company, I. de la Torre Shareholder of: Eli Lilly and Company, Employee of: Eli Lilly and Company, Y. Tanaka Grant/research support from: Mitsubishi-Tanabe, Takeda, Bristol-Myers, Chugai, Astellas, Abbvie, MSD, Daiichi-Sankyo, Pfizer, Kyowa- Kirin, Eisai, Ono, Speakers bureau: Daiichi-Sankyo, Astellas, Pfizer, MitsubishiTanabe, Bristol-Myers, Chugai, YL Biologics, Eli Lilly and Company, Sanofi, Janssen, UCB, E. Feist Grant/research support from: Eli Lilly and Company, Novartis, Roche, BMS, Consultant for: Eli Lilly and Company, Novartis, Roche, Pfizer, Abbvie, BMS, MSD, Sanofi, Speakers bureau: Eli Lilly and Company, Novartis, Roche, Pfizer, Abbvie, BMS, MSD, Sanofi

DOI: 10.1136/annrheumdis-2018-eular.1927

\section{THU0110 EARLY TREATMENT AND LOW DOSE CORTICOSTEROIDS MIGHT DECREASE MORTALITY IN EARLY ARTHRITIS: RESULTS FROM THE RECORD LINKAGE OF CLINICAL AND ADMINISTRATIVE DATABASES}

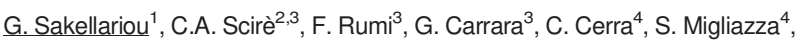
R. Caporali ${ }^{1}$, C. Montecucco $1{ }^{1}$ Chair And Division Of Rheumatology, IRCCS Policlinico San Matteo Foundation, Pavia; ${ }^{2}$ Rheumatology Unit, Department of Medical Sciences, University of Ferrara, Ferrara; ${ }^{3}$ Epidemiology Unit, Italian Society of Rheumatology, Milano; ${ }^{4}$ Department of information and control management, ATS Pavia, Pavia, Italy

Background: In patients with rheumatoid arthritis (RA) increased mortality, especially for cardiovascular (CV) events, is still described, despite the advances in RA management.

Objectives: To evaluate the impact of early diagnosis and treatment with disease modifying anti-rheumatic drugs (DMARDs) on mortality in patients with early RA and undifferentiated arthritis (UA) through record linkage between clinical and administrative databases.

Methods: Consecutive patients with RA or UA from an early arthritis clinic (20052016), treated with tight control to achieve DAS28 <3.2, were included. Health assessment Questionnaire (HAQ) and date of symptom onset were recorded at baseline. Data on mortality, cause of death and drug prescription derived from administrative healthcare databases, linked to the clinical database. Cox regression models were used to evaluate the impact of the interval from symptom onset to diagnosis (categorised $<3$ months, 3-6 months, $>6$ months), from diagnosis to treatment ( $<3$ months, $>3$ months, never) and from onset to treatment $(<3$ months, 3-6 months, $>6$ months, never) on overall mortality. Analyses were adjusted for age, gender, ACPA positivity, Charlson comorbidity index, HAQ and median daily prednisone dose. Results were presented as hazard ratios $(\mathrm{HR})$ with $95 \% \mathrm{Cl}$. Secondary analyses categorising prednisone in low dose ( $\leq 5 \mathrm{mg} / \mathrm{day})$ or medium-high dose ( $>5 \mathrm{mg} /$ day) were performed, as well as analyses evaluating CV mortality as outcome. Moreover, analyses excluding patients not receiving DMARDs and patients dying in the first year of observation were conducted.
Results: A total of 857 patients (62\% RA, 73\% female, median (IQR) age $59,{ }^{47-71}$ mean (sd) baseline DAS28 3.08 (0.97)) were included. After a median (IQR) follow-up of $80^{51-109}$ months, 77 patients died ( 2 in the first year); of the 41 patients with known cause of death, 9 were for $C V$ causes. An interval $>3$ months between diagnosis and introduction of DMARDs or never introducing DMARDs related to higher mortality (table 1). The mean daily prednisone dose was not a significant predictor of mortality, while in all secondary analyses patients receiving low-dose prednisone, compared to those never receiving corticosteroids, had a lower mortality (eg. HR $(95 \% \mathrm{Cl}) 0.45(0.26,0.78)$ in the model including time between symptom onset and diagnosis). Patients not starting DMARDs, compared to these starting within 3 months from diagnosis, had a higher CV mortality), while the intervals between onset and diagnosis and onset and treatment were not significant predictors. Analyses limited to patients receiving DMARDs and with the exclusion of patients dying in the first year yielded to similar results.

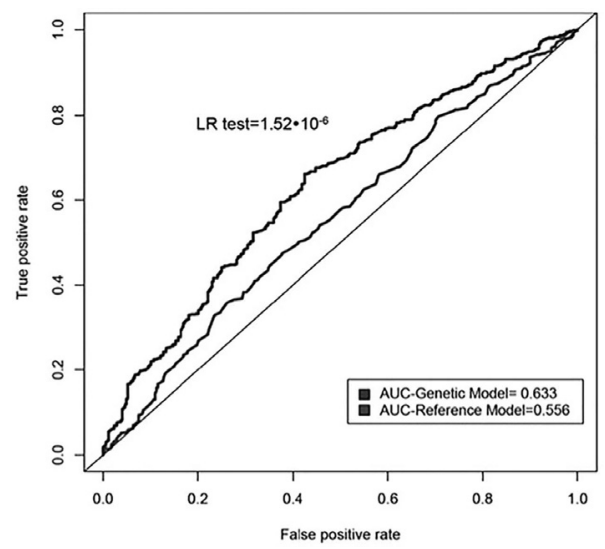

Conclusions: In patients with early RA and UA treatment delay significantly increases mortality, while low-dose corticosteroids seem to decrease mortality. These result support strategies aiming at early access to treatment and the use of low dose corticosteroids in the initial treatment strategy.

Disclosure of Interest: None declared

DOI: 10.1136/annrheumdis-2018-eular.6260

\section{THU0111 FREQUENCY AND PREDICTORS OF SUSTAINED REMISSION IN PATIENTS WITH EARLY RHEUMATOID ARTHRITIS TREATED WITH CONVENTIONAL SYNTHETIC DISEASE MODIFYING DRUGS}

G. Zanframundo, S. Bugatti, A. Manzo, F. Benaglio, G. Sakellariou, C. Montecucco, R. Caporali. Early Arthritis Clinic, Division of Rheumatology, University of Pavia, IRCCS Policlinico San Matteo Foundation, Pavia, Italy

Background: The management of patients with early rheumatoid arthritis (RA) should be aimed at reaching the target of disease remission as soon as possible. In order to prevent joint damage and disability, and eventually allow treatment withdrawal, the state of disease remission should be also maintained. Whilst point remission is frequently achieved in early $R A$, the sustainability of remission in clinical practice remains poorly investigated.

Objectives: To investigate the prevalence and predictors of sustained remission in patients with early RA treated with conventional synthetic disease anti-rheumatic drugs (csDMARDs).

Methods: We evaluated 533 RA patients from the Pavia early arthritis inception cohort not in remission at baseline with at least 24 months of follow-up. Patients had arthritis of short duration ( $<12$ months of symptoms) and were treatment-naïve at presentation. After diagnosis, patients were initiated a treatto-target regimen with methotrexate aiming at low disease activity according to the 28-joint disease activity score (DAS28 $\leq 3.2$ ), and were seen at regular intervals ( 2 months in the first 6 months, then trimestrally). Point remission was defined as the achievement of DAS28 remission $(<2.6)$ or SDAl (simplified disease activity index) remission $(\leq 3.3)$ at any time point within the first 12 months. Sustained remission was defined as mean DAS28 $<2.6$ and mean SDAI $\leq 3.3$ in the 3 visits following first remission. The frequency and predictors of point remission and sustained remission were analysed by Cox and binary regression respectively.

Results: $287 / 533$ (53.9\%) patients achieved point DAS28 remission and 234/ $533(43.9 \%)$ point SDAI remission. Independent predictors of point DAS28 remission were male gender $(\mathrm{HR}[95 \% \mathrm{Cl}] 1.84$ [1.36-2.50]), shorter symptoms' duration ( $\mathrm{HR}[95 \% \mathrm{Cl}] 0.99$ [0.98-0.99], a lower tender joint count at baseline ( $\mathrm{HR}[95 \% \mathrm{Cl}] 0.97$ [0.94-0.99]), better functional status ( $\mathrm{HR}[95 \% \mathrm{Cl}]$ 